\title{
Availability of a remote online hemodynamic monitoring system during treatment in a private dental office for medically high-risk patients
}

\author{
Shinya Yamazaki \\ Hiroyoshi Kawaai \\ Shigeo Sasaki \\ Kazuhiro Shimamura \\ Hiroshi Segawa \\ Takahiro Saito \\ Special Care Department in Dentistry, \\ Ohu University Dental Hospital, \\ Koriyama city, Fukushima prefecture, \\ Japan
}

Correspondence: Shinya Yamazaki 3I-I Misumido Tomita Koriyama,

Fukushima, 963-86II Japan

Tel +8I 249329337

Fax +81249389192

Email zakiyama@ops.dti.ne.jp

\begin{abstract}
The importance of systemic management to prevent accidents is increasing in dentistry because co-morbid illnesses in an aging society and invasive surgical procedures are increasing. In this prefecture, a new medical system called the remote online hemodynamic monitoring system (ROHMs) was started in 2001. Eight private dental offices participated in this trial. When dental practitioners feel the risk of a dental procedure, they can contact via ROHMs to this hospital. Then, the hemodynamic data (blood pressure, heart rate, ECG, $\mathrm{SpO}_{2}$, and RPP) of the patient in the clinic can be transmitted here via the internet, and the images and the voice can be transmitted as well. The availability of this system was assessed in 66 patients (98 cases). The most frequent complications were hypertension, heart disease, and diabetes mellitus. Systemic management included monitoring during the dental procedure (71.4\%), checking vital signs after an interview (15.3\%), and monitoring under sedation $(13.3 \%)$. There were $35.7 \%$ of all cases where an unscheduled procedure was necessary for the systemic management. Based on a questionnaire, the majority of the patients felt relieved and safe. This system creates a situation where a specialist is almost present during the procedure. This system will provide significant assistance for future medical cooperation for risk management.
\end{abstract}

Keywords: online, high-risk patient, dental treatment, medical cooperation, medical accident, risk management

\section{Introduction}

As a result of advanced health care, the current Japanese average life span is 78.53 years old in males, and 85.49 years old in females, and these are highest levels in the world (Dwyer 2005). On the other hand, co-morbid illnesses are increasing with the aging society and advanced health care (Kanemoto et al 2007), and more invasive procedure in dentistry is increasing due to increased use of surgical procedures such as a dental implant technology (Jabero and Sarment 2006). Several fatal accidents during dental procedures have, however, been reported (Davies and Campbell 1990; Burrowes et al 1992; Findler and Galili 2002; Ring 2003; Noguchi et al 2006). Therefore, the importance of systemic management during dental procedure is increasing recently to prevent such accidents (Fukayama and Yagiela 2006). In Ohu University Dental Hospital (OUDH), a new medical cooperation system, called the remote online hemodynamic monitoring system (ROHMs) has been employed since 2001 in a clinical risk management trial for dentistry. When the dental practitioner in a private dental office feels that there are hemodynamic risks to the patient associated with the dental procedure, they can contact OUDH via the internet. Then, the patients' hemodynamic data, an image of the procedure, and the voice of the dental practitioner can be transmitted to 
OUDH. The efficacy of the ROHMs was assessed based on case analyses and patients' questionnaires.

\section{Methods ROHMs}

Figure 1 shows a diagram of ROHMs. This system consists of a central system and terminal system. The central system was installed in the special care department in $\mathrm{OUDH}$, and includes a personal computer with a camera (Hewlett Packard; Vectra VL), an operating system (Microsoft; Windows 98), and application software (Colin Japan; Systemic Management Network System for Dentistry). The terminal system was installed in private dental offices in same prefecture, and this system consists of a TV phone (Titech Japan; SP-1000), and a hemodynamic monitor (Colin Japan; BP-88 Moneo). When dental practitioners in a private dental office feel there are hemodynamic risks associated with the dental procedure, they call the special care department in OUDH. Then, if a staff member of the special care department is available, then they are able to contact each other via the internet by ROHMs. In addition, they can request assistance in managing and monitoring the patient. However, when no staff member of the special care department in OUDH is available, then they need to make an appointment. In ROHMs, the hemodynamic data (NIBP, noninvasive blood pressure; HR, heart rate; ECG, electrocardiogram; $\mathrm{SpO}_{2}$, percutaneous arterial oxygen saturation; RPP, rate pressure product) of the patient in the private dental office can be transmitted by the internet line to the special care department in OUDH, and the image and the voice can be also transmitted simultaneously via the TV phone. The NIBP is measured by a humerus manchette. The ECG is monitored by lead 2. The HR is counted by a plethysmograph or ECG. The RPP is calculated from the product of systolic blood pressure and heart rate, and it correlates to myocardial oxygen consumption (Wilkinson et al 1979). The zoom and view of the camera in the terminal system can be controlled from the central system. The various settings of the monitor, the starting of measurement, and the setting of measurement intervals of the NIBP can be also controlled from the central system. Adjustments of voice register and volume are available from the central system. For the security of the central system, fingerprint identification of the registered dentist is required when the user logs on to control the system. All registered dentists who manage the central system are specialists in dental anesthesia in the special care department.

\section{Participant and analyses}

Eight regular private dental offices participated in this trial. These eight private dental offices were chosen by a conference between the prefecture dental association and OUDH. Two dental offices participated in this trial at the same time because there were only 2 terminal systems. Therefore, one dental office used one terminal system
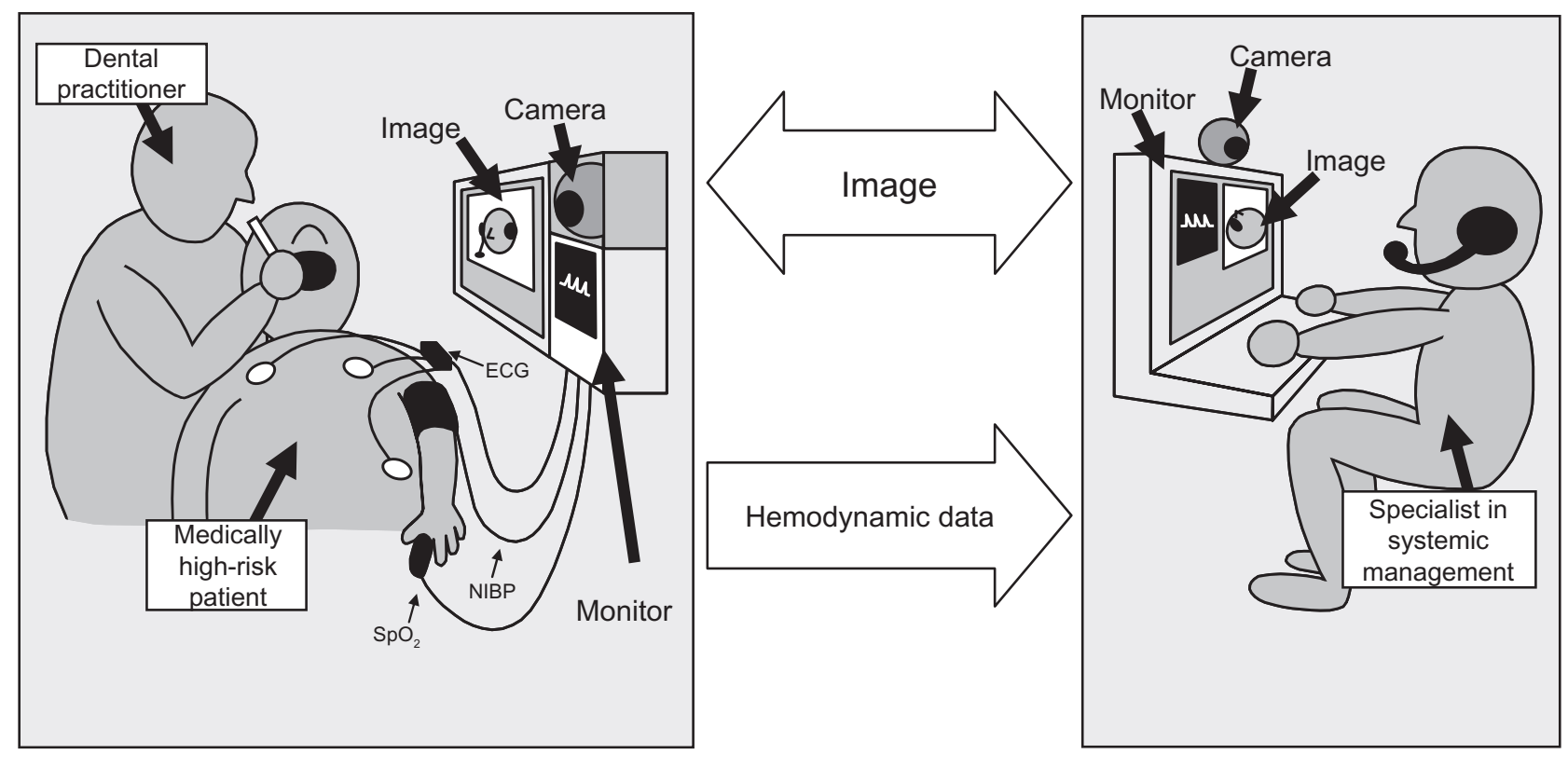

Figure I Diagram of remote online hemodynamic monitoring systems.

Abbreviations: NIBP, noninvasive blood pressure; $\mathrm{HR}$, heart rate; $\mathrm{ECG}$, electrocardiogram; $\mathrm{SpO}_{2}$, percutaneous arterial oxygen saturation. 
for approximately 20 months. All cases of ROHMs were analyzed retrospectively regarding the patients' complications and the management methods. In addition, the results were accessed by questionnaires from the patients who agreed to participate.

\section{Results}

Table 1 shows the background of the cases. From 2001 to 2007, 66 patients (98 cases) were monitored with ROHMs based on the demand from eight private dental offices. Therefore, approximately 1 case was monitored per month. The average age of the patients was $65.8 \pm 14.2$ years old (minimum 22, maximum 87 ). The gender of the patients included 36 males (54.5\%) and 30 females (45.5\%). Table 2 shows the significant patient complications. The most frequent complications were hypertension (28.6\%), heart disease $(27.5 \%)$, diabetes mellitus (10.2\%), and psychiatric disease $(7.1 \%)$. Table 3 shows the systemic management methods. Systemic management included monitoring during the dental procedure $(71.4 \%)$, checking vital signs after an interview (15.3\%), and monitoring under sedation (13.3\%). Table 4 shows the unscheduled procedure for the systemic management. An unscheduled procedure was necessary for the systemic management in $35.7 \%$ of all cases. The unscheduled procedures for the systemic management included consultation with a medical doctor (42.9\%), use of a depressor for hypertension (25.7\%), and discontinuance of dental procedure $(14.3 \%)$. Table 5 shows the results of questionnaires from the patients. The questionnaires were obtained from 30 of 66 patients (collection rate $45.5 \%$ ). The average age of the patients who completed the questionnaire was $64.2 \pm 10.1$ years old (minimum 24, maximum 79), and included 14 males (46.7\%) and 16 females (53.3\%). Most of patients indicated a good opinion such as "It is safe for the patient" and "I was able to relax". However, there were minority opinions such as "I felt embarrassed", and "I felt frightened".

Table I Background of the cases

\begin{tabular}{ll}
\hline Enforcement period & $\begin{array}{l}\text { From 200I to 2007 } \\
\text { (84 months) }\end{array}$ \\
\hline $\begin{array}{l}\text { Number of participation dental } \\
\text { offices }\end{array}$ & 8 \\
Number of total patients & 66 \\
Number of total cases & 98 \\
Number of cases per month & 1.16 \\
Average age of the patients $(\mathrm{yr})$ & $65.8 \pm 14.2$ \\
Minimum - Maximum age $(\mathrm{yr})$ & $22-87$ \\
Gender (male : female) & $36: 30(54.5 \%: 45.5 \%)$ \\
\hline
\end{tabular}

Table 2 Patients' primary complications

\begin{tabular}{|c|c|c|c|}
\hline Complication & Number & Percent & Details \\
\hline Hypertension & 28 & $28.6 \%$ & \\
\hline Cardiac disease & 15 & $15.3 \%$ & $\begin{array}{l}\text { Arrhythmia } 9, \\
\text { Pace maker } 2, \\
\text { Cardiomegaly } 2, \\
\text { Valve disease } 2\end{array}$ \\
\hline Coronary disease & 12 & $12.2 \%$ & $\begin{array}{l}\text { Angina } 7 \text {, Old } \\
\text { myocardial } \\
\text { infarction } 4\end{array}$ \\
\hline Diabetes mellitus & 10 & $10.2 \%$ & \\
\hline Psychiatric disease & 7 & $7.1 \%$ & $\begin{array}{l}\text { Mental retardation 5, } \\
\text { Dementia I, } \\
\text { Schizophrenia I }\end{array}$ \\
\hline Dental phobia & 5 & $5.1 \%$ & \\
\hline Respiratory disease & 4 & $4.1 \%$ & $\begin{array}{l}\text { Asthma 2, COLD I, } \\
\text { Lung cancer I }\end{array}$ \\
\hline Cerebral palsy & 3 & $3.1 \%$ & \\
\hline Drug allergy & 3 & $3.1 \%$ & \\
\hline Post-cerebral stroke & 3 & $3.1 \%$ & $\begin{array}{l}\text { Post-infarction 2, } \\
\text { Post-hemorrhage I }\end{array}$ \\
\hline Renal disease & 2 & $2 \%$ & $\begin{array}{l}\text { Hemodialysis by chronic } \\
\text { renal failure } 2\end{array}$ \\
\hline Liver disease & 2 & $2 \%$ & Liver cirrhosis 2 \\
\hline Muscle disease & 1 & $1 \%$ & Muscular dystrophy I \\
\hline Others & 3 & $3.1 \%$ & \\
\hline Total & 98 & $100 \%$ & \\
\hline
\end{tabular}

\section{Discussion}

\section{High-risk in dentistry}

In Japan, advances in medicine have greatly increased the survival of patients with severe health problems and have significantly prolonged life in elderly individuals with systemic disorders (Dwyer 2005). In fact, in patients 70 years or older, $83.9 \%$ of them had co-morbid illnesses in Japan (Kanemoto et al 2007). As shown in Table 2, hypertension, heart disease, and diabetes mellitus are present in the majority of subjects. Therefore, many fatal accidents during dental procedures have been reported (Ring 2003; Noguchi et al 2006). However, the increasing incidence of fatal accident seems to depend on not only spread of increased co-morbid illnesses in the aging society but also of the increased use of invasive procedures in dentistry. Especially, the implant technology has also resulted in competition between the dental offices, because it brings a significant income to dentists. Recently, a real-time surgical navigation system was developed to provide fully integrated surgical and prosthetic planning for dental implants (Jabero and Sarment 2006). Consequently, the administration of more invasive implant procedures in the dental office will increase further. However, such invasive implant procedures induce rare fatal accidents. Particularly, death due to pulmonary 
Table 3 Systemic management methods

\begin{tabular}{lll}
\hline Systemic management & Number & Percent \\
\hline Monitoring during the dental procedure & 70 & $71.4 \%$ \\
Checking vital signs after an interview & 15 & $15.3 \%$ \\
Monitoring under intravenous sedation & 9 & $9.2 \%$ \\
Monitoring under nitrous oxide sedation & 4 & $4.1 \%$ \\
Total & 98 & $100 \%$ \\
\hline
\end{tabular}

edema and an air embolism can occur during an implant procedure due to surgical stress (Davies and Campbell 1990; Burrowes et al 1992; Findler and Galili 2002). In addition, the administration of local anesthetics and the extensive dental procedures may cause stress and systemic disorders (Fukayama and Yagiela 2006). In order to avoid serious reactions, dentists should be required to continuously monitor the patients' systemic condition (Fukayama and Yagiela 2006). Therefore, the importance of systemic management during dental procedure has recently increased. In almost all university dental hospitals in Japan, specialists in dental anesthesia in the special care department are taking charge of the role of the systemic management. The specialist in systemic management should thus be present next to the procedure dentist during procedures on medically high-risk patients. Therefore, this system is a breakthrough for the dental procedure of medically high-risk patients in private dental offices, because this system puts the specialist right at the dental practitioners' side. A systematic prospective study has not yet been reported except for this study, although a few reports have described the use of this system in Japan. However, a systematic study regarding the availability of this system is considered to be important since this system is expected to rapidly expand and develop in the future.

\section{Appropriate use of ROHMs}

The technology and preparation for emergency resuscitation, venipuncture, and drug administration are different in each dental practitioner and dental office. If there is no preparation to address an emergency accident in a dental office, consideration is necessary to prevent such accidents as much as possible. For that, the most important thing is to understand the patients' systemic condition sufficiently before the dental procedure using a health interview via a $\mathrm{TV}$ phone. If the information is insufficient, it is necessary contact the patients' attending medical doctor concerning the patients' pathological condition. When it is judged that there is some risk in the dental procedure in dental office, the treatment is recommended at the university dental hospital. Presently, the appropriate usage of this system is to prevent the accidents from occurring in the dental clinic. This means that ROHMs should be used for health interviews, diagnosis, and monitoring by specialists at the university dental hospital. Therefore, there were many cases (15.3\%) that ended after only the interview, as shown in Table 3. In addition, Table 4 shows that there are many cases requiring consultation with a medical doctor $(42.9 \%)$, the use of a depressor $(25.7 \%)$, and the discontinuance of dental procedure $(14.3 \%)$. These observations indicate that the system thus performed adequately. We advised the oral administration of the nifedipine for nine cases when the blood pressure increased remarkably, because oral nifedipine has a mild effect, and it does not decrease the blood pressure to an abnormal range. In addition, dental practitioners who administer oral nifedipine tend to be specialists of dental anesthesia and oral surgery. We should therefore select the safest methods depending on each dental practitioner's capacity and our responsibility.

The results of the questionnaires indicated that most patients had a good opinion of the system, noting that it was effective, comforting, and increased their safety. Furthermore, there was no patient who reported a poor impression. However, there were also minority opinions indicating that some resented the lack of privacy and that the monitoring made them nervous. These results suggest that this system can be accepted by the medically high-risk patients. However, it is also necessary to provide sufficient explanation, to avoid making the patient nervous.

\section{Problems and future of ROHMs}

This system provided low (slow) audiovisual quality due to the use of a 256-kbps internet connection. The animation does not provide smooth movement similar to an analog image. Besides, it is often difficult to hear either the practitioner or the specialist. Especially, elderly with poor hearing might not be able to hear the discussion. However, this will be improved in the future with high speed connections using optical fibers and advanced hardware. Then, not only

Table 4 Unscheduled procedures for the systemic management

\begin{tabular}{lll}
\hline Unscheduled procedures & Number & Percent \\
\hline Consultation with a medical doctor & 15 & $15.3 \%$ \\
Use of a depressor for hypertension & 9 & $9.2 \%$ \\
Discontinuance of dental procedure & 5 & $5.1 \%$ \\
Procedure for a shock & 3 & $3.1 \%$ \\
Use of a coronary vasodilator & 2 & $2 \%$ \\
Antibiotics infusion & 1 & $1 \%$ \\
Total & 35 & $35.7 \%$ \\
\hline
\end{tabular}


Table 5 Results of questionnaires from the patients

Obtained questionnaires

Average age of the patients (yr)

Minimum - Maximum age (yr)

Gender (male : female)

\section{$30 / 66$ patients (Collection rate $45.5 \%$ )}

$64.2 \pm 10.1$

24-79

$14: 16(46.7 \%: 53.3 \%)$

Question A.What was your first impression when you hear this system?

\begin{tabular}{llll}
\hline Very good & Good & Neutral & Bad \\
$6(20 \%)$ & $16(53.3 \%)$ & $8(26.7 \%)$ & $0(0 \%)$
\end{tabular}

Question B.What was your impression when you experienced this system?

\begin{tabular}{llll}
\hline Very good & Good & Neutral & Bad \\
I3 (43.3\%) & II $(36.7 \%)$ & $6(20 \%)$ & $0(0 \%)$
\end{tabular}

Question C. How was the reception of university dental hospital?

\begin{tabular}{llll}
\hline Very good & Good & Neutral & Bad \\
$9(30 \%)$ & $18(60 \%)$ & $3(10 \%)$ & $0(0 \%)$
\end{tabular}

$9(30 \%)$

$0(0 \%)$

$0(0 \%)$

Question D. How was the safety impression degree about this system?

\begin{tabular}{llll}
\hline Very good & Good & Neutral & Bad \\
$18(60 \%)$ & $7(23.3 \%)$ & $5(16.7 \%)$ & $0(0 \%)$
\end{tabular}

Question E. How do you feel if this system develops in future?

\begin{tabular}{llll}
\hline $\begin{array}{l}\text { Very good } \\
\text { II }(36.7 \%)\end{array}$ & Good & Neutral & Bad \\
I7 (56.7\%) & $2(6.7 \%)$ & $0(0 \%)$ \\
Question F. Could you write other opinion about this system? & $0(0 \%)$ & I felt \\
\hline No answer & It is safe & It was difficult to & embarrassed \\
I9 (63.3\%) & $5(16.7 \%)$ & $2(6.7 \%)$ & $2(6.7 \%)$ \\
\hline
\end{tabular}

systemic management by high-quality communication but also image (X-ray, local condition, sentences, etc.) analysis will be possible in a remote situation.

In fact, there was no instance of a staff shortage regarding the usage of OUDH, because 2 or 3 dental anesthetists are always available per case of general anesthesia or sedation in OUDH. However, if many private dental offices use this system in same time, it is obvious there will be insufficient manpower at university to accommodate them. Furthermore, that would be costly to the university. However, dental practitioners receiving assistance in the management of high-risk patients from the university dental hospital can thus be increased by this system. In fact, several high-risk patients from the dental practitioners were accepted thanks to the use of this system. Using this system, the dental practitioner will be easily able to treat the high-risk patient who in the past could not be treated at a local dental office. On the other hand, this system will require an increased staff and increased cost to the patient and or the dental practitioner in the future, because the need for the systemic management for the medically high-risk patient in dentistry has risen remarkably in Japan.
More advanced technical support cannot be provided in this system because the specialists are not on the site. At least for now, the ideal usage of this system should be for the health interview, diagnosis, and monitoring of the patients' systemic condition before and during the dental procedure, because there is a limit in a remote support now. However, a more advanced system will one day allow the specialist to be virtually at the side of the practitioner, because the online remote-controlled range will surely be wider in the future.

This system still has some problems that need to be worked out at the present stage. However, they are expected to be solved in the future based on the results of further medical cooperation.

\section{Conclusions}

This system is a breakthrough for the dental procedure of medically high-risk patients in a private dental office because this system allows the specialist to monitor the patients during the procedure. The patients had a good impression of this system. The current problems will be improved in the future and this system should therefore be able to provide 
significant assistance in the future medical cooperation for risk management.

\section{Disclosure}

The authors report no conflicts of interest.

\section{References}

Burrowes P, Wallace C, Davies JM, et al. 1992. Pulmonary edema as a radiologic manifestation of venous air embolism secondary to dental implant surgery. Chest, 101:561-2.

Davies JM, Campbell LA. 1990. Fatal air embolism during dental implant surgery: a report of three cases. Can J Anaesth, 37:112-21.

Dwyer J. 2005. Global health and justice. Bioethics, 19:460-75.
Findler M, Galili D. 2002. Cardiac arrest in dental offices. Report of six cases. Refuat Hapeh Vehashinayim, 19:79-87.

Fukayama H, Yagiela JA. 2006. Monitoring of vital signs during dental care. Int Dent $J, 56: 102-8$.

Jabero M, Sarment DP. 2006. Advanced surgical guidance technology. Implant Dent, 15:135-42.

Kanemoto K, Satoh H, Ishikawa H, et al. 2007. Co-morbid illnesses in patients with respiratory disease. Tuberk Toraks, 55:127-34.

Noguchi I, Ohno H, Takano K, et al. 2006. Fatal hyperthermia due to dental treatment. Oral Surg Oral Med Oral Pathol Oral Radiol Endod, 101:61-4.

Ring ME. 2003. Terrible accidents during tooth extraction in the earlier days of dentistry. $N$ Y State Dent $J, 69: 31-4$.

Wilkinson PL, Moyers JR, Ports T, et al. 1979. Rate-pressure product and myocardial oxygen consumption during surgery for coronary artery bypass. Circulation, 60:170-3. 